\title{
An accurate and efficient scheme for acoustic-structure interaction problems based on unstructured mesh
}

\author{
X.Y. Cui ${ }^{1,2, *}$, X. Hu ${ }^{1,2}$, G. Wang ${ }^{1,2}$, G.Y. $\mathrm{Li}^{1,2}$ \\ ${ }^{1}$ State Key Laboratory of Advanced Design and Manufacturing for Vehicle Body, Hunan University, \\ Changsha, 410082, PR China \\ ${ }^{2}$ Collaborative Innovation Center for Intelligent New Energy Vehicle, Shanghai, 200092, PR China
}

\begin{abstract}
This paper focuses on the accurate and efficient numerical implementation of acoustic-structure coupling formulations using the edge-based smoothed finite element method for the flexible shell and the gradient-weighted finite element method for the acoustic fluid field, namely, the ES/GW-FEM. The shell is discretized using the simplest linear triangular elements and the edge-based smoothing domain is then constructed. By introducing an edge local coordinate system, the edge-based smoothing operation is performed on the smoothing domain. As for the acoustic fluid domain, the tetrahedron elements are adopted. A compacted support domain is then constructed and the gradient weighted operation is performed on the support domain. To model the exterior acoustic domain, an artificial boundary is introduced and the Dirichlet-to-Neumann (DtN) boundary condition is imposed. Based on the appropriate compatibility and equilibrium conditions on the interface boundaries, the coupled ES/GW-FEM formulation is finally obtained. Both the interior acoustic-structure coupled problems and exterior acoustic-structure coupled problems are available as the DtN boundary is considered. Numerical examples demonstrate that the coupled ES/GW-FEM achieves much higher accuracy and works more reliable compared with the coupled FEM/FEM in solving practical engineering problems.
\end{abstract}

Keywords: Acoustic; shell structure; acoustic-structure interaction; numerical methods; the edge-based smoothed finite element method (ES-FEM); the gradient-weighted finite element method (GW-FEM).

* Corresponding author. Tel: 86-731-8821717; Fax: 86-731-8822051

Email address: cuixy@hnu.edu.cn (X.Y. Cui) 


\begin{tabular}{|c|c|}
\hline \multicolumn{2}{|l|}{ Nomenclature } \\
\hline$a_{n}^{j}$ & coefficient related to the DtN boundary condition \\
\hline$A_{k}, A_{k l}$ & area of the smoothing domain and the sub-domain $l$ \\
\hline$\hat{\mathbf{B}}_{m}, \hat{\mathbf{B}}_{b}, \hat{\mathbf{B}}_{s}$ & $\begin{array}{l}\text { strain matrix of the membrane strain, bending strain and shear strain } \\
\text { in the element local coordinate system }\end{array}$ \\
\hline $\mathbf{B}_{i}^{e}$ & acoustic gradient matrix \\
\hline$\overline{\mathbf{B}}_{f}^{k}$ & effective acoustic gradient matrix of element $k$ \\
\hline$c$ & speed of the acoustic wave \\
\hline $\begin{array}{l}\mathbf{D}_{m}, \mathbf{D}_{b}, \mathbf{D}_{s} \\
\mathbf{f}\end{array}$ & $\begin{array}{l}\text { material matrices corresponding to the membrane deformation, } \\
\text { bending deformation and shear deformation } \\
\text { the distributed load applied on the shell }\end{array}$ \\
\hline $\mathbf{F}_{f}, \mathbf{F}_{s}$ & system force vector of the acoustic fluid and shell \\
\hline$F_{n}^{j}$ & polynomials in trigonometric functions \\
\hline$h_{n}^{(2)}$ & spherical Hankel function of the second kind \\
\hline $\mathrm{j}$ & unit imaginary number \\
\hline$k$ & wave number \\
\hline $\mathbf{K}_{A B}^{b}$ & system stiffness matrix related to the DtN boundary condition \\
\hline$\overline{\mathbf{K}}_{f}$ & weighted system stiffness matrix of the fluid \\
\hline$\overline{\mathbf{K}}_{s}$ & smoothed stiffness matrix of the shell \\
\hline $\mathbf{L}$ & spatial coupling matrix \\
\hline $\mathbf{m}$ & material matrices related to the mass matrix \\
\hline$M$ & DtN operator \\
\hline$M_{k}$ & a set that contains all the nodes in the support domain \\
\hline $\mathbf{M}_{f}, \mathbf{M}_{s}$ & system mass matrix of the fluid and shell \\
\hline n & normal vector along the acoustic fluid boundary \\
\hline$\hat{\mathbf{N}}$ & the FEM shape functions in the element local coordinate system \\
\hline $\mathbf{N}_{f}$ & generalized FEM shape functions in the fluid domain \\
\hline$n c$ & total element number of the support domain \\
\hline$N_{\text {edge }}$ & number of the edges of the entire background mesh \\
\hline$N_{i}(\mathbf{x})$ & linear FEM shape function values \\
\hline$N_{i^{\prime} x}, N_{i^{\prime} y}, N_{i^{\prime} z}$ & partial derivative of the shape function \\
\hline$N_{p}$ & number of nodes in each element \\
\hline $\mathbf{n}_{s}, \mathbf{n}_{f}$ & $\begin{array}{l}\text { normal vector along the shell structure and the acoustic fluid } \\
\text { boundary }\end{array}$ \\
\hline$p$ & acoustic pressure \\
\hline $\mathbf{P}$ & acoustic pressure vector \\
\hline$P_{n}^{j}$ & associated Legendre function of the first kind \\
\hline$\widehat{\mathbf{p}}_{f}^{k}$ & effective acoustic pressure vector \\
\hline$r$ & radial direction \\
\hline
\end{tabular}




\begin{tabular}{|c|c|}
\hline$\hat{\mathbf{R}}_{m}, \hat{\mathbf{R}}_{b}, \hat{\mathbf{R}}_{s}$ & $\begin{array}{l}\text { transformation matrices from the local element system to the global } \\
\text { one }\end{array}$ \\
\hline$\overline{\mathbf{R}}_{m}, \overline{\mathbf{R}}_{b}, \overline{\mathbf{R}}_{s}$ & $\begin{array}{l}\text { transformation matrices from the global system to the edge-based } \\
\text { system }\end{array}$ \\
\hline$t$ & time \\
\hline$\tilde{t}$ & thickness of the shell \\
\hline$\hat{\mathbf{T}}$ & $\begin{array}{l}\text { transformation matrix of the nodal displacement vectors from the } \\
\text { global element system to the local vectors }\end{array}$ \\
\hline $\mathbf{u}$ & displacement field \\
\hline$\hat{\mathbf{u}}$ & generalized displacement in the element local coordinate system \\
\hline$\hat{u}, \hat{v}, \hat{w}$ & three translational displacements of the element coordinate system \\
\hline$\ddot{\mathbf{u}}_{s}$ & normal acceleration of the shell \\
\hline$\ddot{u}_{f}$ & normal acceleration component of the acoustic fluid \\
\hline$u_{s}, u_{f}$ & displacements of the structure and the acoustic fluid particle \\
\hline$v$ & particle velocity \\
\hline$v_{k}^{j}$ & particle velocity of the $j$-th element \\
\hline $\bar{v}_{k}$ & weighted velocity of the master element $k$ \\
\hline$V_{j}$ & the volume of the $j$-th element \\
\hline$w_{j}$ & weight function values \\
\hline $\bar{x}, \bar{y}, \bar{z}$ & edge-based local coordinate system \\
\hline$\hat{\gamma}_{x z}, \hat{\gamma}_{x z}$ & components of the shear strain \\
\hline$\Gamma_{B}$ & artificial boundary \\
\hline$\Gamma_{f}$ & rigid boundary \\
\hline$\hat{\varepsilon}_{m}, \hat{\varepsilon}_{b}, \hat{\varepsilon}_{s}$ & $\begin{array}{l}\text { membrane strain, bending strain and shear strain in the element local } \\
\text { coordinate system }\end{array}$ \\
\hline $\bar{\varepsilon}_{m}, \bar{\varepsilon}_{b}, \bar{\varepsilon}_{s}$ & $\begin{array}{l}\text { smoothed membrane strain, bending strain and the shear strain in the } \\
\text { edge-based local coordinate system }\end{array}$ \\
\hline$\hat{\theta}_{x} \hat{\theta}_{y}$ & two rotational displacements in the element local coordinate system \\
\hline$\rho, \rho_{f}$ & density of the shell and the acoustic fluid \\
\hline$\sigma_{s \mid n}$ & structural stress \\
\hline$\tau$ & traction imposed on the natural boundary \\
\hline$\Phi_{j}$ & global shape function values \\
\hline$\chi_{j}$ & defined weighting coefficient \\
\hline$\omega$ & angular frequency \\
\hline$\Omega_{f}, \Omega_{s}$ & acoustic fluid domain and the flexible structure domain \\
\hline$\Omega_{k}$ & smoothing domain of an edge $k$ \\
\hline$\partial \Omega_{s f}$ & the coupling interface \\
\hline
\end{tabular}




\section{Introduction}

Thin-walled structures are commonly used as covering parts of mechanical products, such as the automobile, airplane, train, steamer, and so on. The acoustic performances of the closed cavities covered by such structures are always of great concern as these cavities are directly related to passengers. As the mutual influence between the thin structures and acoustic fluid cannot be neglected, such problems are typical acoustic-structural coupling problems. Nowadays, there are growing concerns about the acoustic performances of such cavities and more and more resources are devoted to improving the performances. The experimental way was the most used approach during the development process in the past decades; however, it takes far longer and costs far more. With the development of the computer application technology, numerical simulation has gradually become an effective and popular way to solve the acoustic-structure coupled problems.

The finite element method (FEM) [1-2] and the boundary element method (BEM) [3-4] are both the well-developed and widely used numerical method in engineering analysis. The FEM is famous for its simplicity, efficiency and adaptability. These fascinating characteristics guarantee that the FEM can occupy a very important position in simulating the dynamic behavior of the structure, acoustic and acoustic-structure interaction problems. The BEM, another numerical method that is often adopted in analyzing acoustic-structural problems, is mainly used for the acoustic part especially when the acoustic fluid filed is unbounded. That is because the Sommerfeld condition is automatically satisfied in the BEM and thus there is no special treatment to the exterior domain is needed.

Though the coupled FEM/FEM [5-8] and FEM/BEM [9-13] have already been used in analyzing acoustic-structural problems, some inherent drawbacks seriously restrict the application of the methods. The coupled FEM/FEM is available at lower frequencies; however, the accuracy of the coupled FEM/FEM has left a lot to be desired when the frequency is higher. To improve the calculation accuracy, many numerical treatments have been proposed [14-16]. When the coupled FEM/BEM is 
employed, the fluid domain only requires meshing on the field boundary and thus fewer elements are generated compared with the coupled FEM/FEM model. However, the coupled FEM/BEM model also suffers from the fully populated matrices and leads to a heavy burden in both memory consumption and computing time [17]. To solve the problem, the finite element/fast multipole boundary element (FE/FMBE) [18] coupling formulation and other improved methods [19-22] have been employed to analyze acoustic-structure coupled problems. Though such inherent drawbacks are got settlement to some extent, there are still some imperfections that need to be improved when extending these methods to practical engineering applications.

It has been proved that the standard FEM model suffers from the "overly-stiff” property and thus results in a significant loss of computation accuracy and reliability [23-25]. In simulating acoustic problems, the "overly-stiff” FEM model makes the numerical speed of the acoustic wave larger than the real one and finally leads to the well-known numerical dispersion. The dispersion error increases dramatically with the increasing frequency [26], hence, the coupled FEM/FEM is powerless when the desired frequency is higher. Therefore, it is of great important to develop a discrete model with proper "softened" stiffness to improve the computation accuracy. For the sake of this, a strain smoothing method was proposed by Chen et al. [27-28], and a generalized gradient smoothing (GGS) technique [29-31] has been further formulated by Liu and his co-workers. Based on the generalized gradient smoothing technique, a serious of smoothed finite element methods such as the cell-based smoothed finite element method (CS-FEM) [32-34], the node-based smoothed finite element method (NS-FEM) [35-38], the edge-based smoothed finite element method (ES-FEM) [39-42], the face-based smoothed finite element method (FS-FEM) [43-44] and so on [45-47] have been developed and widely used. Among these smoothed finite element method, the NS-FEM and the ES-FEM are two "start" elements and possess many excellent features. The NS-FEM performs very well in adaptive analysis and feature analysis [35]. Unfortunately, the NS-FEM cannot be used to solve dynamic problems directly for the NS-FEM model exhibits "overly-soft” which will lead to temporal instability [30-31]. The ES-FEM encounters the same instability problems in 
one-dimensional; however, the ES-FEM is found to be stable and provides much more accurate results in solving 2D and 3D problems [41]. Studies [41] have proved that the ES-FEM exhibits neither "overly-stiff” nor "overly-soft” behaviors and can hence achieve so-called “close-to-exact” system stiffness. Recently, based on the basic theory of the generalized gradient smoothing technique, the gradient-weighted finite element method (GW-FEM) for acoustic simulation has been proposed by Wang and his co-workers [48]. In this method, the calculation precision can be improved greatly with limited increase of computational effort by weighting the acoustic gradient of the connected element simply.

As the ES-FEM shows enormous advantages in simulating plate and shells problems with the commonly used unstructured mesh [40], it is quite a promising formulation to look forward in acoustic-structural coupling analysis. Another, but no less important, aspect revolves around acoustic-structural coupling analysis is the computation of the acoustic field. The GW-FEM improves the calculation accuracy of the acoustic field significantly while keeping high computational efficiency. Thus, the GW-FEM is an effective solver for complex acoustic problems. Besides, the acoustic-structure coupled problem is a complex process that involves the modeling of acoustic fluid domain, structure domain and as well as the interactions between the fluid and structure [49]. Thus, to obtain reliable numerical solutions, the correct choice of the coupling model is essential beyond the proper models for the structure and acoustic fluid. As the feedback of the acoustic pressure onto the thin-walled structures cannot be neglected, the so-called one-way coupling [50] is unsuitable and the appropriate coupling scheme $[10,11,44]$ is needed.

This paper focuses on the accurate and efficient numerical implementation of acoustic-structure coupling formulation using the edge-based smoothed finite element method for the flexible shell and the gradient weighted finite element method for the acoustic fluid field, namely, the ES/GW-FEM. The ES-FEM formulation based on the first order shear deformation theory is conducted to model the shell structure and the discrete shear gap method (DSG) [51] is introduced to eliminate the shear-locking phenomenon. The simplest linear triangular elements are employed to discrete the 
flexile shell and the edge-based smoothing domain is further constructed based on the background elements. Then the system stiffness matrix is finally obtained based on the edge-based smoothing domain. As for the acoustic fluid, linear tetrahedral elements are employed. Based on the obtained elements, a compacted support domain is then constructed and the gradient weighting operation is carried out in the support domain. To model the exterior acoustic-structural problems, the well-known Dirichlet-to-Neumann (DtN) boundary condition [52-56] is introduced to truncate the infinite domain. Using the appropriate compatibility and equilibrium conditions on the interface boundaries, the coupled ES/GW-FEM formulation is finally obtained. Because of the superior performances of the ES-FEM and GW-FEM, the coupled ES/GW-FEM is expected to improve the accuracy and stability significantly.

The paper is organized as follows. The basic formulations of ES-FEM for shell structure and GW-FEM for acoustic fluid is presented in the next section. After that, the coupled formulation is formed in section 3. In section 4, the performances of the coupled ES/GW-FEM are investigated through several examples. Finally, the paper is summarized with some concluding remarks.

\section{Structural and acoustic simulation}

A typical acoustic-structure coupled problem is illustrated in Fig. 1. For interior acoustic-structural problems, the acoustic cavity is enclosed by the flexible structure $\Omega_{s}$ and the rigid boundary $\Gamma_{f}$. When it comes to exterior acoustic-structure coupled problem, the flexible structure $\Omega_{s}$ and the rigid boundary $\Gamma_{f}$ forms the inner boundary and an artificial boundary $\Gamma_{B}$ is introduced to truncate the infinite exterior acoustic domain. To obtain a coupled formulation, the theoretical bases of the ES-FEM for the structure and GW-FEM for the acoustic field are introduced briefly.

\subsection{Edge-based strain smoothing operation for shell structures}

\subsubsection{Basic equations for shell structures}

Consider a thin-walled structure as part of the structure-acoustic coupling domain. 
Shell elements are employed to model the structures for its simplicity and efficiency. By choosing the middle surface of the shell as the reference plane, the shell structure domain is further discretized into a set of flat shell elements. The field variables of the shell element in the element local coordinate system are defined as three translational displacements $\hat{u}, \hat{v}, \hat{w}$ and two rotational displacements $\hat{\theta}_{x}, \hat{\theta}_{y}$ (the symbol '^’' stands for the element local coordinate system).

The first order shear deformation theory is employed here, and thus the generalized strains can be written in terms of the mid-plane deformations

$$
\hat{\varepsilon}_{m}=\left\{\begin{array}{c}
\frac{\partial \hat{u}_{0}}{\partial \hat{x}} \\
\frac{\partial \hat{v}_{0}}{\partial \hat{y}} \\
\frac{\partial \hat{u}_{0}}{\partial \hat{y}}+\frac{\partial \hat{v}_{0}}{\partial \hat{x}}
\end{array}\right\} \quad \hat{\varepsilon}_{b}=\left\{\begin{array}{c}
\frac{\partial \hat{\theta}_{y}}{\partial \hat{x}} \\
-\frac{\partial \hat{\theta}_{x}}{\partial \hat{y}} \\
\frac{\partial \hat{\theta}_{y}}{\partial \hat{y}}-\frac{\partial \hat{\theta}_{x}}{\partial \hat{x}}
\end{array}\right\} \quad \hat{\varepsilon}_{s}=\left\{\begin{array}{c}
\frac{\partial \hat{w}_{0}}{\partial \hat{x}}+\hat{\theta}_{y} \\
\frac{\partial \hat{w}_{0}}{\partial \hat{y}}-\hat{\theta}_{x}
\end{array}\right\}
$$

where $\hat{\varepsilon}_{m}, \hat{\varepsilon}_{b}, \hat{\varepsilon}_{s}$ are the three parts of the generalized strains which represent the membrane strain, bending strain and transverse shear strain, respectively.

By applying the principle of virtual work, the weak-form of the dynamic variation equation can be obtained

$$
\begin{gathered}
\int_{\Omega_{s}}\left(\delta \varepsilon_{m}^{\mathrm{T}} \mathbf{D}_{m} \varepsilon_{m}+\delta \varepsilon_{b}^{\mathrm{T}} \mathbf{D}_{b} \varepsilon_{b}+\delta \varepsilon_{s}^{\mathrm{T}} \mathbf{D}_{s} \varepsilon_{s}\right) \mathrm{d} \Omega+\int_{\Omega_{s}} \delta \mathbf{u}^{\mathrm{T}} \mathbf{m} \ddot{\mathbf{u}} \Omega \\
-\int_{\Omega_{s}} \delta \mathbf{u}^{\mathrm{T}} \mathbf{f} \mathrm{d} \Omega-\int_{\Gamma_{s}} \delta \mathbf{u}^{\mathrm{T}} \tau \mathrm{d} \Gamma=0
\end{gathered}
$$

where $\mathbf{D}_{m}, \mathbf{D}_{b}$ and $\mathbf{D}_{s}$ represent the material matrices corresponding to the membrane deformation, bending deformation and shear deformation; $\mathbf{f}$ is the distributed load applied on the shell; $\tau$ is the traction imposed on the natural boundary; $\mathbf{m}$ is a matrix which has the form of

$$
\mathbf{m}=\operatorname{diag}\left\{\rho \tilde{t}, \rho \tilde{t}, \rho \tilde{t}, \rho \tilde{t}^{3} / 12, \rho \tilde{t}^{3} / 12,0\right\}
$$

in which $\rho$ and $\tilde{t}$ represent the density and the thickness of the shell, respectively. The first three terms of the matrix $\mathbf{m}$ are related to the three translational degrees of freedom and the final three are corresponded to rotational degrees of freedom. As the 
Reissner-Mindlin hypothesis is employed in this paper, the moment of inertia corresponded to the last term is zero. Thus, the last term in Eq. (3) is zero.

\subsubsection{Edge-based strain smoothing operation}

In a triangular shell element, the generalized displacement can be obtained based on the linear shape function, which has the form of

$$
\hat{\mathbf{u}}=\sum_{i=1}^{3} \hat{\mathbf{N}}_{i}(\mathbf{x}) \hat{\mathbf{u}}_{i}
$$

where $\hat{\mathbf{u}}$ represents the generalized displacement field of a node in the element; $\hat{\mathbf{N}}_{i}$ and $\hat{\mathbf{u}}_{i}$ are the shape function value and the nodal displacement of node $i$.

By substituting Eq. (4) into Eq. (1), the expression of the membrane strain and the bending strain can be further obtained

$$
\hat{\boldsymbol{\varepsilon}}_{m}=\sum_{i=1}^{3} \hat{\mathbf{B}}_{m i} \hat{\mathbf{u}}_{i}, \quad \hat{\boldsymbol{\varepsilon}}_{b}=\sum_{i=1}^{3} \hat{\mathbf{B}}_{b i} \hat{\mathbf{u}}_{i}
$$

To eliminate the shear-locking, the shear strain is obtained by adopting the "Discrete Shear Gap” (DSG) method [51]. Then the shear strain in each element can be written as

$$
\hat{\boldsymbol{\varepsilon}}_{s}=\left\{\begin{array}{l}
\hat{\gamma}_{x z} \\
\hat{\gamma}_{y z}
\end{array}\right\}=\hat{\mathbf{B}}_{s} \hat{\mathbf{u}}=\sum_{i=1}^{3} \hat{\mathbf{B}}_{s i} \hat{\mathbf{u}}_{i}
$$

In order to carry out the edge-based strain smoothing operation, the structure domain is further subdivided into $N_{\text {edge }}$ non-overlapping and non-gap sub-domains based on the background mesh, in which $N_{\text {edge }}$ denotes the number of the edges of the entire background mesh. Each sub-domain is an edge-based smoothing domain and the detailed process of how to construct the edge-based smoothing domain is briefly illustrated in Fig. 2. For an interior edge $k$, the smoothing domain $\Omega_{k}$ is constructed by connecting the nodes of the edge and the centroids of the elements sharing the same edge. As for the edge located on the boundary, the smoothing domain is a single sub-domain, and thus the strain matrix is the same as that of FEM. 
For the shell problem, the local coordinate system is defined based on each element. As the smoothing domain $\Omega_{k}$ of an interior edge $k$ is composed of two sub-domains located at two different elements, the strain smoothing operation cannot be performed directly. For the sake of solving this problem, an edge-based local coordinate system $(\bar{x}, \bar{y}, \bar{z})$ is further defined, as shown in Fig. 2. In the edge-based local coordinate system, $\bar{X}$ is defined to be coinciding with the edge $k, \bar{Z}$ is decided by the average normal direction of the two elements associated to the edge, $\bar{y}$ is obtained from the cross product of $\bar{x}$ and $\bar{Z}$. Based on the simple transformation rules for each element, the smoothed strains in the edge-based local coordinate system can be further written as

$$
\begin{aligned}
\overline{\boldsymbol{\varepsilon}}_{m} & =\overline{\mathbf{R}}_{m} \hat{\mathbf{R}}_{m} \hat{\boldsymbol{\varepsilon}}_{m} \\
\overline{\boldsymbol{\varepsilon}}_{b} & =\overline{\mathbf{R}}_{b} \hat{\mathbf{R}}_{b} \hat{\boldsymbol{\varepsilon}}_{b} \\
\overline{\boldsymbol{\varepsilon}}_{s} & =\overline{\mathbf{R}}_{s} \hat{\mathbf{R}}_{s} \hat{\boldsymbol{\varepsilon}}_{s}
\end{aligned}
$$

where $\overline{\boldsymbol{\varepsilon}}_{m}, \overline{\boldsymbol{\varepsilon}}_{b}$ and $\overline{\boldsymbol{\varepsilon}}_{s}$ denote the membrane strain, bending strain and the transverse shear strain in the smoothing domain; $\overline{\mathbf{R}}_{m}, \overline{\mathbf{R}}_{b}$ and $\overline{\mathbf{R}}_{s}$ represent the transformation matrices that link the strains from the global coordinate system to the edge-based coordinate; $\hat{\mathbf{R}}_{m}, \hat{\mathbf{R}}_{b}$ and $\hat{\mathbf{R}}_{s}$ represent the transformation matrices from the local element system to the global one. Space prevents that discussion here, but the readers are referred to Ref $[40,44]$ for the detailed expressions of the transformation matrices.

For an arbitrary smoothing domain $\Omega_{k}$, there should be two sub-domains $\Omega_{k l}$ $(l=1,2)$ for the interior edge and one sub-domain $\Omega_{k l}(l=1)$ for the boundary edge. The smoothed strains over the smoothing domain in the edge-based local coordinate system can be obtained with weighted average for the compatible strains are constants in each element. After the strain smoothing operation, the smoothed strains can be further written as 


$$
\begin{aligned}
& \overline{\boldsymbol{\varepsilon}}_{m}^{(k)}=\frac{1}{A_{k}} \sum_{l} A_{k l} \overline{\boldsymbol{\varepsilon}}_{m}^{(k l)} \\
& \overline{\boldsymbol{\varepsilon}}_{b}^{(k)}=\frac{1}{A_{k}} \sum_{l} A_{k l} \overline{\boldsymbol{\varepsilon}}_{b}^{(k l)} \\
& \overline{\boldsymbol{\varepsilon}}_{s}^{(k)}=\frac{1}{A_{k}} \sum_{l} A_{k l} \overline{\boldsymbol{\varepsilon}}_{s}^{(k l)}
\end{aligned}
$$

where $\overline{\boldsymbol{\varepsilon}}_{m}^{(k l)}, \overline{\boldsymbol{\varepsilon}}_{b}^{(k l)}$ and $\overline{\boldsymbol{\varepsilon}}_{s}^{(k l)}$ represent the corresponding strains of the sub-domain; $A_{k}$ denotes the area of the smoothing domain; $A_{k l}$ denotes the area of the sub-domain.

Substituting Eqs. (5), (6), (7) into Eq. (8), the smoothed strains can be finally obtained and written as

$$
\begin{aligned}
\overline{\boldsymbol{\varepsilon}}_{m}^{(k)} & =\overline{\mathbf{B}}_{m}^{(k)} \mathbf{u}^{(k)}=\frac{1}{A_{k}} \sum_{l} A_{k l} \overline{\mathbf{R}}_{m}^{(k)} \hat{\mathbf{R}}_{m}^{(k l)} \hat{\mathbf{B}}_{m}^{(k l)} \hat{\mathbf{T}}^{(l)} \\
\overline{\boldsymbol{\varepsilon}}_{b}^{(k)} & =\overline{\mathbf{B}}_{b}^{(k)} \mathbf{u}^{(k)}=\frac{1}{A_{k}} \sum_{l} A_{k l} \overline{\mathbf{R}}_{b}^{(k)} \hat{\mathbf{R}}_{b}^{(k l)} \hat{\mathbf{B}}_{b}^{(k l)} \hat{\mathbf{T}}^{(l)} \\
\overline{\boldsymbol{\varepsilon}}_{s}^{(k)} & =\overline{\mathbf{B}}_{s}^{(k)} \mathbf{u}^{(k)}=\frac{1}{A_{k}} \sum_{l} A_{k l} \overline{\mathbf{R}}_{s}^{(k)} \hat{\mathbf{R}}_{s}^{(k l)} \hat{\mathbf{B}}_{s}^{(k l)} \hat{\mathbf{T}}^{(l)}
\end{aligned}
$$

where $\mathbf{u}^{(k)}$ is a vector that contains all the nodes of the elements sharing the edge $k$, $\hat{\mathbf{T}}$ is the transformation matrix that translates the nodal displacement vectors $\mathbf{u}_{i}$ from the global element system to the local vectors $\hat{\mathbf{u}}_{i}$.

Based on the smoothed strains shown in Eq. (9) and the interpolation form in Eq. (4), the weak-form of the dynamic variation equation can be written in the discrete form

$$
\overline{\mathbf{K}}_{s} \mathbf{u}_{s}+\mathbf{M}_{s} \ddot{\mathbf{u}}_{s}=\mathbf{F}_{s}
$$

in which $\overline{\mathbf{K}}_{s}$ denotes the smoothed stiffness matrix

$$
\overline{\mathbf{K}}_{s}=\sum_{k=1}^{N_{\text {edge }}} \overline{\mathbf{K}}_{s}^{(k)}
$$

with the sub-matrix has the form of

$$
\overline{\mathbf{K}}_{s}^{(k)}=\left(\overline{\mathbf{B}}_{m}^{(k)}\right)^{\mathrm{T}} \mathbf{D}_{m} \overline{\mathbf{B}}_{m}^{(k)} A_{k}+\left(\overline{\mathbf{B}}_{b}^{(k)}\right)^{\mathrm{T}} \mathbf{D}_{b} \overline{\mathbf{B}}_{b}^{(k)} A_{k}+\left(\overline{\mathbf{B}}_{s}^{(k)}\right)^{\mathrm{T}} \mathbf{D}_{s} \overline{\mathbf{B}}_{s}^{(k)} A_{k}
$$

$\mathbf{M}_{s}$ is the system mass matrix

$$
\mathbf{M}_{s}=\int_{\Omega_{s}} \mathbf{N}^{\mathrm{T}} \mathbf{m} \mathbf{N d} \Omega
$$


$\mathbf{F}_{s}$ denotes the system force vector

$$
\mathbf{F}_{s}=\int_{\Omega_{s}} \mathbf{N}_{s}^{\mathrm{T}} \mathbf{f} \mathrm{d} \Omega+\int_{\Gamma_{s}} \mathbf{N}_{s}^{\mathrm{T}} \tau \mathrm{d} \Gamma
$$

\subsection{Gradient weighted operation for the acoustic fluid}

\subsubsection{Basic equations for acoustic problem}

In general, the computational acoustic can be classified into two classes: the interior acoustic problems and the exterior acoustic problems. No matter which class of problems is taken into account, the acoustic wave propagates in the ideal fluid can be governed by the following Helmholtz equation

$$
\Delta p-\frac{1}{c^{2}} \frac{\partial^{2} p}{\partial t^{2}}=0
$$

where $\Delta$ represents the Laplace operator, $p$ is the acoustic pressure, $c$ denotes the speed of the acoustic wave and $t$ denotes time. At the coupling interface of the structure and acoustic field, the momentum balance require that

$$
\frac{\partial p}{\partial n}=-\rho_{f} \ddot{u}_{f}
$$

in which $n$ represents the boundary surface normal vector of the acoustic domain, the symbols $\rho_{f}$ and $\ddot{u}_{f}$ respectively denote the density of the fluid and the normal acceleration component of the fluid at the coupling interface $\partial \Omega_{s f}$. The other boundary of the acoustic fluid domain is the rigid wall boundary, which has the form of

$$
\frac{\partial p}{\partial n}=0
$$

As the computational domain is finite, these equations are enough to model interior acoustic problems. When it comes to exterior acoustic problems, however, these equations are necessary but not sufficient for the computational domain is infinite. In simulating exterior problems, to assure the uniqueness of the solution, the well-known Sommerfeld radiation condition should be taken into account. The Sommerfeld radiation condition can be expressed as 


$$
\lim _{r \rightarrow \infty} r^{\frac{d-1}{2}}\left(\frac{\partial p}{\partial r}+\mathrm{j} k p\right)=0
$$

in which, $\mathrm{j}$ represents the imaginary unit.

Though Eq. (18) ensures the uniqueness of the solution, the problem is still cannot be solved by the common domain based numerical methods such as the FEM. To solve the exterior acoustic problems, an artificial boundary $\Gamma_{B}$ is introduced to truncate the infinite domain and the well-known Dirichlet-to-Neumann (DtN) boundary condition [52] is imposed on the artificial boundary. Once the artificial boundary is introduced, the original infinite computational domain is divided into two equivalent problems, one located in the finite domain $\Omega_{f}$ and the other one located in the infinite domain $\Omega_{\text {inf }}$, as shown in Fig. 1 . The latter problem can be solved analytically according to Keller and Givoli’s study [52] and only the problem located in the finite domain need to be solved. In general, the artificial boundary can be chosen as a circle or ellipse for two dimensional cases and a sphere [52] or ellipsoid for three dimensional cases [56]. For the convenience of obtaining the analytical solution, the artificial boundary is constructed as a sphere centered at the origin in this paper.

The DtN map relates the pressure and velocity data on $\Gamma_{B}$ and possesses the general form of

$$
\frac{\partial p}{\partial n}=-M p \quad \text { on } \quad \Gamma_{B}
$$

where $M$ represents the DtN operator. The implementation of Eq. (19) can be easily done based on the explicit expressions of the DtN operator. In three dimensions, the normal derivative of the acoustic pressure on the artificial boundary can be easily obtained in the spherical coordinates

$$
p_{v}(R, \theta, \phi)=\left.\frac{\partial p(r, \theta, \phi)}{\partial n}\right|_{r=R}=-\sum_{n=0}^{\infty} \int_{\Gamma_{B}} m_{n}\left(\theta, \phi, \theta^{\prime}, \phi^{\prime}\right) p\left(R, \theta^{\prime}, \phi^{\prime}\right) \mathrm{d} \Gamma
$$

where $R$ represents the radius of the artificial boundary and $m_{n}\left(\theta, \phi, \theta^{\prime}, \phi^{\prime}\right)$ represents 


$$
\begin{gathered}
m_{n}\left(\theta, \phi, \theta^{\prime}, \phi^{\prime}\right)=\sum_{j=0}^{n}{ }^{\prime} \beta_{j n} P_{n}^{j}(\cos \phi) P_{n}^{j}\left(\cos \phi^{\prime}\right) \cos j\left(\theta-\theta^{\prime}\right) \\
\beta_{j n}=-\frac{(2 n+1)(n-j) !}{2 \pi R^{2}(n+j) !} \frac{k h_{n}^{(2)^{\prime}}(k R)}{h_{n}^{(2)}(k R)}
\end{gathered}
$$

in which, $P_{n}^{j}$ denotes the associated Legendre function of the first kind; $h_{n}^{(2)}$ is the spherical Hankel function of the second kind.

Based on the equations above, the standard Galerkin weak-form of the Helmholtz equation can be obtained

$$
\frac{1}{c^{2}} \int_{\Omega_{f}} \delta p \cdot \frac{\partial^{2} p}{\partial t^{2}} \mathrm{~d} \Omega_{f}-\int_{\Omega_{f}} \delta \nabla p \cdot \nabla p \mathrm{~d} \Omega_{f}+\rho_{f} \int_{\partial \Omega_{s f}} \delta p \cdot \ddot{u}_{f} \mathrm{~d} \Gamma-\int_{\Gamma_{B}} \delta p \cdot M \cdot p \mathrm{~d} \Gamma=0
$$

in which the last term is only for exterior acoustic problems. By introducing the artificial boundary, the boundary value problems in infinite domain can be solved using the domain based methods equivalently.

\subsubsection{Fundamental of the gradient weighted operation}

To carry out the gradient weighted operation, the problem domain is discretized into $N_{e}$ linear tetrahedral elements. Tetrahedral elements are preferred in discretizing complex geometries in engineering and the discretization process can be easily done using any mesh generator automatically. Once the linear tetrahedral elements are obtained, the acoustic pressure at any point in an element is interpolated using the nodal pressure. The interpolation is processed based on the linear shape function, which has the form of

$$
p=\sum_{i=1}^{N p} N_{i}(\mathbf{x}) p_{i}=\mathbf{N} \mathbf{p}
$$

where $N_{p}$ represents the number of nodes in each element; $N_{i}(\mathbf{x})$ and $p_{i}$ denote the linear FEM shape function values and the unknown nodal pressure, respectively.

In the ideal fluid, the gradient of acoustic pressure $p$ and the particle velocity $v$ can be linked together through the following equation

$$
\nabla p+\mathrm{j} \rho_{f} \omega v=0
$$


By substituting Eq. (24) into Eq. (25), the velocity that relates to the field gradient can be obtained in each element

$$
\mathbf{v}^{e}=-\frac{1}{j \rho_{f} \omega} \sum_{i=1}^{N p} \mathbf{B}_{i}^{e} p_{i}
$$

where

$$
\mathbf{B}_{i}^{e}=\left[\begin{array}{lll}
N_{i^{\prime} x} & N_{i^{\prime} y} & N_{i^{\prime} z}
\end{array}\right]^{\mathrm{T}}
$$

In the formulation of the GW-FEM, the field gradient is further weighted based on the element itself together with its adjacent elements sharing common faces. In order to maintain clarity and simplicity, the center element $\Omega_{k}$ is defined as the master element and its adjacent elements are defined as the slave elements. As illustrated in Fig. 3, for a given element $k$, the total connected domains formed a support domain of the element and labeled as $\Omega_{k}^{s}$. The weighted acoustic pressure can be obtained from the weighted velocity through Eq. (12). Thus the weighted velocity of the master element $\Omega_{k}$ can be written as

$$
\bar{v}_{k}=\sum_{j=1}^{n c} \Phi_{j} v_{k}^{j}
$$

where $n c$ and $v_{k}^{j}$ represent the total element number of the support domain and the particle velocity of the $j$-th element, respectively; $\Phi_{j}$ is the global shape function values of the $j$-th element. The global shape function represents the relationship between the elements in a support domain and has the form of

$$
\Phi_{j}=\frac{w_{j}}{\sum_{i}^{n c} w_{i}}
$$

in which, $w_{j}$ is the weight function of the $j$-th element which is defined based on the element volume

$$
w_{j}=\chi_{j} V_{j}
$$

where $V_{j}$ is the volume of the $j$-th element; $\chi_{j}$ is the defined weighting coefficient

$$
\chi_{j}= \begin{cases}2.0 & \text { master element } \\ 1.0 & \text { slave elements }\end{cases}
$$


More concrete design and detailed analysis on the global shape function can be found in Ref [47]. Besides, it's clear that the defined global shape function satisfies the partition of unity. Based on the obtained global shape function, the weighted velocity in Eq. (28) can be further written as

$$
\bar{v}_{k}=-\frac{1}{\mathrm{j} \rho_{f} \omega} \sum_{j=1}^{n c}\left[\Phi_{j} \sum_{i=1}^{N p} \mathbf{B}_{i}^{j} p_{i}^{j}\right]
$$

where $\mathbf{B}_{i}^{j}$ and $p_{i}^{j}$ represent the $\mathbf{B}_{i}$ and $p_{i}$ values in the $j$-th element. Then the weighted acoustic gradient of the master element is finally obtained

$$
\nabla \bar{p}_{k}=\sum_{I \in M_{k}} \overline{\mathbf{B}}_{I} p_{I}=\overline{\mathbf{B}}_{f}^{k} \widehat{\mathbf{p}}_{f}^{k}
$$

in which $M_{k}$ denotes a set that contains all the nodes in the support domain; $\overline{\mathbf{B}}_{f}^{k}$ and $\hat{\mathbf{p}}_{f}^{k}$ represent the effective gradient matrix and the effective acoustic pressure vector.

Then substituting Eq. (24) and Eq. (33) into the weak-form shown in Eq. (23), the discretized system equations can then be obtained and written in the following matrix form

$$
\left(\overline{\mathbf{K}}_{f}+\mathbf{K}_{A B}^{b}\right) \mathbf{p}+\mathbf{M}_{f} \ddot{\mathbf{p}}=\mathbf{F}_{f}
$$

where $\overline{\mathbf{K}}_{f}$ denotes the weighted system stiffness matrix

$$
\overline{\mathbf{K}}_{f}=\sum_{k=1}^{N_{e}} \overline{\mathbf{K}}_{f}^{k}
$$

in which the sub-matrix $\overline{\mathbf{K}}^{k}$ has the form of

$$
\overline{\mathbf{K}}^{k}=\int_{\Omega_{k}}\left(\overline{\mathbf{B}}_{f}^{k}\right)^{\mathrm{T}}\left(\overline{\mathbf{B}}_{f}^{k}\right) \mathrm{d} \Omega=\left(\overline{\mathbf{B}}_{f}^{k}\right)^{\mathrm{T}}\left(\overline{\mathbf{B}}_{f}^{k}\right) V_{k}
$$

$\mathbf{M}_{f}$ denotes the system mass matrix

$$
\mathbf{M}_{f}=\frac{1}{c^{2}} \int_{\Omega_{f}} \mathbf{N}_{f}^{\mathrm{T}} \mathbf{N}_{f} \mathrm{~d} \Omega_{f}
$$

$\mathbf{F}_{f}$ denotes the acoustic force vector

$$
\mathbf{F}_{f}=-\rho_{f} \int_{\partial \Omega_{s f}} \mathbf{N}_{f}^{\mathrm{T}} \ddot{\mathbf{u}}_{f} \mathrm{~d} \Gamma
$$

$\mathbf{P}$ denotes the acoustic pressure vector

$$
\{\mathbf{P}\}^{\mathrm{T}}=\left\{p_{1}, p_{2}, \ldots, p_{n}\right\}
$$


$\mathbf{K}_{A B}^{b}$ is the system stiffness matrix related to the DtN boundary condition which can be obtained based on Eq. (20)

$$
\mathbf{K}_{A B}^{b}=\int_{\Gamma_{B}} N_{A}(\mathbf{x}) M N_{B}(\mathbf{x}) \mathrm{d} \Gamma
$$

where $N_{A}$ and $N_{B}$ are the FEM shape functions of any two nodes $A$ and $B$ located on the artificial boundary.

$$
\begin{aligned}
\mathbf{K}_{A B}^{b} & =\int_{\Gamma_{B}} N_{A}(\mathbf{x}) M N_{B}(\mathbf{x}) \mathrm{d} \Gamma \\
& =\sum_{n=0}^{\infty} \sum_{j=0}^{n} a_{n}^{j}\left(\int_{\Gamma_{B}} N_{A}(\mathbf{x}) F_{n}^{j}(\mathbf{x}) \mathrm{d} \Gamma\right)\left(\int_{\Gamma_{B}} N_{B}(\mathbf{x}) F_{n}^{j}(\mathbf{x}) \mathrm{d} \Gamma\right)
\end{aligned}
$$

where $a_{n}^{j}$ is the coefficient which contains the expressions involving the spherical Hankel functions shown in Eq.(22); $F_{n}^{j}$ denotes the polynomials in trigonometric functions which has the form of

$$
F_{n}^{j}=\left[P_{n}^{j}(\cos \phi) \cos j \theta \quad P_{n}^{j}(\cos \phi) \sin j \theta\right]
$$

Ref [51-52] show more parameter information and computational aspects of the DtN boundary condition.

\section{Coupled formulation for acoustic-structural analysis}

Based on the presented formulations for the shell structure and the acoustic fluid, the general coupled acoustic-structural formulation is further derived. To ensure the continuity between the fluid particle and the shell structure during the interaction, the following requirement should be fulfilled at the coupling surface

$$
u_{s} \mathbf{n}=u_{f} \mathbf{n}
$$

where $u_{s}$ and $u_{f}$ are the displacements of the structure and the acoustic fluid particle contacting the structure; $\mathbf{n}$ is the normal vector along the acoustic fluid boundary. Note that $\mathbf{n}$ is defined as the normal vector along the fluid boundary, thus the direction of the normal vector along the shell structure $\mathbf{n}_{s}$ and the direction of the normal vector along the acoustic fluid boundary $\mathbf{n}_{f}$ satisfy the following condition

$$
\mathbf{n}=\mathbf{n}_{f}=-\mathbf{n}_{s}
$$

At the interface, the continuity condition between the structural stress and the 
acoustic pressure should also be fulfilled

$$
\sigma_{s \mid n}=-p
$$

Then the fluid force loading on the shell structure can be obtained

$$
\mathbf{F}_{s}=\int_{\partial \Omega_{s f}} \mathbf{N}_{s}^{\mathrm{T}} \mathbf{n}_{s} \sigma_{s} \mathrm{~d} \Gamma=\int_{\partial \Omega_{s f}} \mathbf{N}_{s}^{\mathrm{T}} \mathbf{n}_{f} p \mathrm{~d} \Gamma=\left(\int_{\partial \Omega_{s f}} \mathbf{N}_{s}^{\mathrm{T}} \mathbf{n}_{f} \mathbf{N}_{f} \mathrm{~d} \Gamma\right) \mathbf{p}
$$

The force from the structure loading on the acoustic fluid can be derived and written as

$$
\mathbf{F}_{f}=-\rho \int_{\partial \Omega_{s f}} \mathbf{N}_{f}^{\mathrm{T}} \ddot{u} f \mathrm{~d} \Gamma=-\rho \int_{\partial \Omega_{s f}} \mathbf{N}_{f}^{\mathrm{T}} \ddot{u_{s}} \mathrm{~d} \Gamma=-\rho\left(\int_{\partial \Omega_{s f}} \mathbf{N}_{f}^{\mathrm{T}} \mathbf{n}_{f} \mathbf{N}_{s} \mathrm{~d} \Gamma\right) \ddot{\mathbf{u}}_{s}
$$

The coupling force vector presented above can be further written as

$$
\begin{gathered}
\mathbf{F}_{s}=\mathbf{L} \mathbf{p} \\
\mathbf{F}_{f}=-\rho_{f} \mathbf{L}^{\mathrm{T}} \ddot{\mathbf{u}}_{s}
\end{gathered}
$$

with the spatial coupling matrix which has the form of

$$
\mathbf{L}=\int_{\partial \Omega_{s f}} \mathbf{N}_{s}^{\mathrm{T}} \mathbf{n}_{f} \mathbf{N}_{f} \mathrm{~d} \Gamma
$$

Based on the equations, the acoustic-structural problem can be finally described by an unsymmetrical system of equations

$$
\left[\begin{array}{cc}
\mathbf{M}_{s} & \mathbf{0} \\
\rho_{f} \mathbf{L}^{T} & \mathbf{M}_{f}
\end{array}\right]\left\{\begin{array}{c}
\ddot{\mathbf{u}}_{s} \\
\ddot{\mathbf{p}}
\end{array}\right\}+\left[\begin{array}{cc}
\overline{\mathbf{K}}_{s} & -\mathbf{L} \\
0 & \overline{\mathbf{K}}_{f}
\end{array}\right]\left\{\begin{array}{c}
\mathbf{u}_{s} \\
\mathbf{p}
\end{array}\right\}=\left\{\begin{array}{c}
\mathbf{F}_{s} \\
\mathbf{F}_{f}
\end{array}\right\}
$$

As the displacement and pressure are all assumed to be time harmonic in the following analysis, the forced frequency response analysis equation for the acoustic-structural problems can be further formulated as

$$
\left[\begin{array}{cc}
\overline{\mathbf{K}}_{s}-\omega^{2} \mathbf{M}_{s} & -\mathbf{L} \\
\rho_{f} \omega^{2} \mathbf{L}^{T} & \overline{\mathbf{K}}_{f}-\omega^{2} \mathbf{M}_{f}
\end{array}\right]\left\{\begin{array}{l}
\mathbf{u}_{s} \\
\mathbf{p}
\end{array}\right\}=\left\{\begin{array}{l}
\mathbf{F}_{s} \\
\mathbf{F}_{f}
\end{array}\right\}
$$

\section{Numerical examples}

In this section, three different types of acoustic-structure coupled numerical examples are employed to verify the effectiveness of the presented ES/GW-FEM formulation. The first two numerical examples are the coupled systems of shell structure and interior acoustic field, including a testing example and a complex engineering example. Besides, a coupled system of shell structure and exterior 
acoustic field is also adopted based on a more complex and large scale light rail model. Three-node triangular elements and four-node tetrahedron elements are employed to discretize the shell and the acoustic field for they can be easily generated for any models with complex geometries. For comparison, the results obtained using the coupled FEM/FEM based on the same mesh are also provided.

\subsection{Flexible shell backed by a closed acoustic cavity}

As shown in Fig. 4, consider an acoustic cavity which is bounded by a flexible shell on the top and rigid walls of other boundaries. The geometrical shape and dimension parameters are labeled in the figure. The flexible shell is made of steel whose material properties are Young's modulus E=210 GPa, Poisson's radio $v=0.3$ and density $\rho_{s}=7800 \mathrm{~kg} / \mathrm{m}^{3}$, with a thickness of $1 \mathrm{~mm}$. The cavity is filled with air whose density is $\rho_{s}=1.225 \mathrm{~kg} / \mathrm{m}^{3}$, and the speed of sound travel in the air is taken to be $c=340 \mathrm{~m} / \mathrm{s}$. The model is discretized into 638 nodes for the shell structure and 2616 nodes for the acoustic field. As there are no analytical solutions for the problems with complicated configurations, numerical results obtained using FEM/FEM with a very fine mesh (5,313 nodes for the shell and 44,898 nodes for the acoustic field) are adopted as the reference.

The natural eigenfrequencies of the flexible shell and the acoustic cavity is studied firstly to investigate the performances of the ES-FEM for shell structure and GW-FEM for acoustic field. Table 1 shows the first 15 non-rigid eigenfrequencies of the shell obtained using the ES-FEM together with the results obtained using FEM and DSG. From the table, it's clear that (1) The ES-FEM is very accurate compared with the standard FEM and DSG. The maximum relative error of the FEM in the first 15 eigenfrequencies is up to $4.801 \%$, which is nearly two times bigger than the maximum relative error of the ES-FEM. (2) The relative errors of the numerical results obtained using the ES-FEM, the standard FEM and DSG all increase with the increase of the mode orders, but the ES-FEM is much more reliable with slow growth. (3) The eigenfrequencies obtained using the standard FEM are all much larger than 
the reference results, while the results of the ES-FEM are smaller and more accurate. This manifests that the standard FEM suffers from the “overly-stiff” phenomenon and the edge-based smoothing operation provides a much softer stiffness. Thus, the ES-FEM is quite a promising formulation to look forward in acoustic-structural coupling analysis.

The first 15 eigenfrequencies of the uncoupled acoustic cavity obtained using both the GW-FEM and the standard FEM is shown in Table 2. From the table, the following conclusions can be generalized. (1) The relative errors of results of both methods increase with the increase of the mode orders; however, the results obtained using the standard FEM deteriorates faster. (2) The eigenfrequencies obtained using the FEM are larger than the reference results, while the results of the GW-FEM are smaller than the reference results and much more accurate. This proves again that the standard FEM suffers from the “overly-stiff” phenomenon.

Then the coupled cavity eigenfrequencies are further investigated using the coupled ES/GW-FEM and the coupled FEM/FEM. The meshes used here is same to the previous and the first 15 eigenfrequencies are listed in Table 3. As shown in the table, (1) the presented coupled ES/GW-FEM formulation can generally provide more accurate results compared with the coupled FEM/FEM and DSG/FEM. The maximum relative error of the coupled eigenfrequencies obtained using the coupled FEM/FEM is up to $3.924 \%$. However, the relative error of the presented method is only $1.602 \%$, two times less than the coupled FEM/FEM. (2) All the first 15 eigenfrequencies calculated using the coupled FEM/FEM and DSG/FEM are larger than the reference which indicates that the coupled FEM/FEM and DSG/FEM both suffers from the “overly-stiff” phenomenon. And as we expected, the coupled ES/GW-FEM provides a properly softened stiffness and results in very accurate results.

As the coupled ES/GW-FEM formulation provides much accurate results for the eigenfrequencies analysis, the forced frequency response analysis (ref $=2 \times 10^{-5} \mathrm{~Pa}$ ) is then conducted to further investigate the coupled ES/GW-FEM. To carry out the procedure, a point time harmonic load of $1.0 \mathrm{~N}$ is subjected at the center of the shell surface. Based on the meshes used in the previous analysis, the forced frequency 
response analysis is conducted. The central point of the shell and two points in the acoustic field are chosen as the concerned points and the responses results of the three points are plotted in Figs. 5-7, respectively. From the figures, it’s clear that (1) all the three coupled methods can provide very accurate results when frequencies is under $400 \mathrm{~Hz}$. (2) With the increase of frequency, the results obtained using the coupled FEM/FEM and DSG/FEM deviate from the reference results quickly; however, the coupled ES/GW-FEM can still accurately predict the distribution tends with small deviation. (3) The coupled FEM/FEM and DSG/FEM behave similarly and only available at low frequency range. (4) When it comes to high frequency range such as over $800 \mathrm{~Hz}$, all the three methods can no longer predict the eigenfrequencies accurately; however, the result obtained using the coupled ES/GW-FEM is still much more reliable. Thus, the presented coupled ES/GW-FEM formulation is very promising in acoustic-structural coupling analysis.

In engineering, the convergence and efficiency are always of great concern. And with the same precision, the most time-efficient method is preferred. Thus, the computational efficiency of the present formulation is further investigated in this subsection. To process the simulation, a global error indicator is defined as

$$
\mathrm{Ge}=\sqrt{\frac{\int_{\Omega}\left(\tilde{v}^{\text {ref }}-\tilde{v}^{h}\right)^{\mathrm{T}}\left(v^{\text {ref }}-v^{h}\right) \mathrm{d} \Omega}{\int_{\Omega}\left(\tilde{v}^{r e f} \cdot v^{r e f}\right) \mathrm{d} \Omega}}
$$

where the symbol " $\sim$ " denotes the complex conjugate; the superscript "ref" and " $h$ " denote the reference solution and the numerical solution, respectively. Then the problem domain is discretized using four different mesh densities (1323, 1995, 2616 and 3883 nodes, respectively). All of the programs of the three different methods are executed based on the same software and hardware environments. The relationship between the CPU time and the global relative error of the coupled FEM/FEM, DSG/FEM and ES/GW-FEM are shown in Fig. 8. As depicted in the figure, the efficiency curve of the coupled ES/GW-FEM is always located on the lower left side of the curves obtained using the coupled FEM/FEM and DSG/FEM, which indicates that the ES/GW-FEM can always produces higher computational efficiency. As to the 
convergence rate, the coupled FEM/FEM and DSG/FEM behave similar with the convergence rate of -0.674 and -0.600 respectively. However, the convergence rate of the present ES/GW-FEM reached to -0.966. These conclusions demonstrate that the coupled ES/GW-FEM is much more efficient than the coupled FEM/FEM and DSG/FEM. Thus, it is a quite promising formulation to look forward in engineering.

\subsection{Passenger compartment with flexible roof panel}

Shell structures are commonly used as covering parts of mechanical products, such as the automobile, airplane, steamer, and so on. Thus, the acoustic-structural coupled problems are often encountered in the development of such products. As the acoustical quality is a very important indicator for such closed cavity, the coupling problem between the mainly covering parts and the passenger compartment cavity is very noteworthy. Thus, in this section, a scale model of coupled passenger compartment system is taken into account. In order to control and reduce the vehicle body's vibration as well as reduce the acoustic responses purposefully, a numerical method which is accurate for predicting field distribution is essential. Therefore, the coupled ES/GW-FEM is further investigated based on the model.

The coupled system is shown in Fig. 9, the roof panel is made of steel whose material properties are same to the previous. The thickness of the roof panel is $1 \mathrm{~mm}$. The passenger compartment cavity is filled with air and the material properties are not repeated here. The roof panel is discretized into 1,674 triangular elements with 896 nodes, and the acoustic field is discretized into 55,773 tetrahedron elements with 12,170 nodes. The numerical results obtained using FEM/FEM with a very fine mesh (4,119 nodes for the shell and 149,724 nodes for the acoustic field) are employed as the reference.

The eigenfrequencies of the coupled passenger compartment system are investigated firstly. The first 15 non-rigid eigenfrequencies obtained using the three coupled methods and together with the reference solutions are listed in Table 4. Similar conclusions can be summarized, (1) the maximum relative error of the coupled eigenfrequencies obtained using the coupled FEM/FEM is up to 5.721\%, 
which is nearly three times larger than maximum relative error of the coupled ES/GW-FEM solutions. (2) The coupled FEM/FEM and DSG/FEM behave similarly and are both low-accuracy. However, the present coupled ES/GW-FEM formulation is more stable and can generally provide more accurate results. (3) Though the eigenfrequencies obtained using both methods are larger than the reference solutions, the results of the coupled ES/GW-FEM are more accurate compared with the coupled FEM/FEM and DSG/FEM. This indicates that the presented formulation is no longer suffers from the “overly-stiff” phenomenon and can be used for analyzing practical engineering problems.

As the forced frequency response analysis provides sufficient information about the coupled eigenfrequencies, the process is conducted for the coupled passenger compartment cavity system. A point time harmonic force of $1.0 \mathrm{~N}$ is imposed at the center of the shell surface. The acoustic response measured at the center of the shell is extracted and plotted in Fig. 9. Besides, the driver's ear point and the back row crew member's ear point are also chosen as the concerned points in the acoustic field. Figs. 11-12 show the acoustic responses of the two concerned points in the acoustic field. The range of the frequency is set from 1 to $400 \mathrm{~Hz}$, which is a great concern in designing a vehicle. As is shown in the figures, (1) all the three methods can obtain reasonable results at lower frequencies. (2) With the increase of frequency, the results obtained using both methods deviate from the reference results; however, the coupled ES/GW-FEM can still accurately predict the distribution tends with very small deviation. The conclusions once again proved that the presented coupled ES/GW-FEM formulation is very promising in analyzing practical engineering acoustic-structure coupled problems.

\subsection{Radiated acoustic field of a light trail carriage}

Nowadays, with the car ownership in the big cities going through roof and the subsequent traffic congestion, rail transport has never attracted so much attention. Subway is one of the most common urban rail transit modes and has been in service for many years. However, the light trail, which is more cost-efficient and convenient, 
is drawn even more attention recently. Many big cities have already built some light trail lines and some of the lines even span the neighborhoods. Thus, research on the radiated acoustic field of the light trail is necessary. To study the radiated acoustic field of the light trail, a scale simplified model is established, as shown in Fig. 13. The covering parts are simplified into shell structure with a thickness of $2 \mathrm{~mm}$ and the internal structure is ignored. Time harmonic excitations are imposed at the junctions between the wheel suspensions and the light trail carriage to simulate the vibration during the light trail running.

To process the simulation, an artificial boundary is introduced to obtain a finite computational domain for the radiated field is infinite. The well-known DtN boundary condition is imposed on the artificial boundary to ensure the equivalence. And based on the introduced artificial boundary, four points are chosen to be the concerned points. The concerned points are located at the right front, right rear, left rear and left front of the running direction of the light train, respectively. As the model is more complex and large scale compared with the other two examples, the model is more persuasive in proving the prospects of the present coupled ES/GW-FEM.

The forced frequency response analysis is conducted for the exterior acoustic structure coupled problem. The shell structure is discretized into 16,622 triangular elements and the acoustic field is discretized into 202,196 tetrahedron elements. As the model is much complex, the reference solutions are obtained using Virtual.lab with very fine mesh (143,352 elements for the shell and 1,697,461 tetrahedron elements for the acoustic field). The frequency responses results of the four different concerned points are depicted in Figs. 14-17, respectively. As shown in the figure, (1) at lower frequencies, all the coupled methods can provide accurate results and keep in good agreement with the reference solutions. (2) With the increasing of the frequency in the whole frequency range, the results obtained using the coupled ES/GW-FEM can still keep in good agreements with the reference solutions, while the results obtained using the coupled FEM/FEM and DSG/FEM deviate from the reference results quickly. Thus the coupled ES/GW-FEM is still valid for the complex structure and exterior acoustic field coupled problems. 


\section{Conclusions}

In this work, a coupled ES/GW-FEM formulation is presented for both interior acoustic-structure coupled problems and exterior acoustic-structure coupled problems. The shell structure is modeled using the ES-FEM based on the simplest linear triangular elements. As for the acoustic fluid field, the linear tetrahedron elements are adopted and the GW-FEM formulation is then conducted. Based on the continuity conditions at the interface between the shell and acoustic field, the coupled ES/GW-FEM formulation is finally obtained. Through a series of numerical examples, the following conclusions can be summarized out:

(1) The coupled ES/GW-FEM is formulated based on the simplest linear triangular elements and tetrahedron elements, and thus the method is very suitable for acoustic-structure coupled problems with complex geometric properties.

(2) Owning to the proper softening effects introduced by the edge-based smoothing operation, the eigenfrequencies of the shell can be predicted precisely using the ES-FEM. Besides, the GW-FEM can predict the eigenfrequencies of the acoustic field accurately, too. And as was expected, through the coupled ES/GW-FEM, the eigenfrequencies of the coupled system can be obtained precisely.

(3) Compared with the classical coupled FEM/FEM and DSG/FEM, the present coupled ES/GW-FEM achieves highest computational efficiency.

(4) The coupled ES/GW-FEM is suitable and effective for both interior acoustic-structure coupled problems and exterior acoustic-structure coupled problems as exemplified by Figures 10-12 and 14-17.

(5) For the analysis of the complex engineering problems, the coupled ES/GW-FEM performs much better and works more reliable compared with coupled FEM/FEM and DSG/FEM. Thus the present method has great application values in solving practical engineering problems.

\section{Acknowledgments}

The support of National Science Foundation of China (11472101), State Key Program 
of National Natural Science of China (61232014) and Hunan Provincial Natural Science Foundation of China (2015JJ3037) are gratefully acknowledged.

\section{References}

[1] O.C. Zienkiewicz, R.L. Taylor (Eds), The Finite Element Method: Its Basis and Fundamentals, fifth ed, Butterworth-Heinemann: Oxford, 2000.

[2] I. Harari, A survey of finite element methods for time-harmonic acoustics, Comput. Methods Appl. Mech. Engrg. 195 (2) (2006) 1594-1607.

[3] T.W. Wu, Boundary element in acoustics: formulations and computer codes, WIT Press, Southampton, 2000.

[4] J.T. Chen, Recent development of dual BEM in acoustic problems, Comput. Methods Appl. Mech. Engrg. 188 (2000) 833-845.

[5] D. J. Neffske, J. A. Wolf, L. J. Howell, Structural-acoustic finite element analysis of the automobile passenger compartment: a review of current practice, J. Sound Vib. 80(2) (1982) 247-66.

[6] S. H. Sung, D. J. Nefske, A coupled structural-acoustic finite element model for vehicle interior noise analysis, J. Vib Acoust. 106 (2) (1984) 314-8.

[7] K. J. Bathe, C. Natikitpaiboon, X. Wang, A mixed displacement-based finite element formulation for acoustic fluid-structure interaction, Comput. Struct. 56 (1995) 225-237.

[8] G. C. Everstine, Finite element formulations of structural acoustics problems, Comput. Struct. 65(3) (1997) 307-321.

[9] G. C. Everstine, F. M. Henderson, Coupled finite element/boundary element approach for fluid structure interaction, J. Acoust. Soc. Am. 87 (1990) 1938-47.

[10] R. A. Jeans, J. C. Mathews, Solution of fluid-structure interaction problems using a coupled finite element and variational boundary element technique, J. Acoust. Soc. Am. 88 (1990) 2459-2466.

[11] Z. S. Chen, G. Hofstetter, H. A. Mang, A Galerkin-type BE-FE formulation for elasto-acoustic coupling. Comput. Methods Appl. Mech. Engrg. 152 (1998) 147-155.

[12] H. D. Ju, S. B. Lee. Multi-domain structural-acoustic coupling analysis using the finite element and boundary element techniques, J. Mech. Sci. Technol. 15(5) (2001) 555-61.

[13] D. Fritze, S. Marburg, H. J. Hardtke. FEM-BEM-coupling and structural-acoustic sensitivity analysis for shell geometries, Comput. Struct. 83 (2005) 143-54.

[14] K. L. Hong, J. Kim, Analysis of free vibration of structural-acoustic coupled systems, partI: development and verification of the procedure, J. Sound Vib. 188(4) (1995) 561-75.

[15] S. M. Kim, M. J. Brennan, A compact matrix formulation using the impedance and mobility approach for the analysis of structural-acoustic systems, J. Sound Vib. 223(1) (1999) 97-113.

[16] R. J. Bernhard, J. E. Huff, Structural-acoustic design at high frequency using the energy finite element method, J. Vib. Acoust. 121 (1999) 295-301.

[17] D. Brunner, M. Junge, L. Gaul, A comparison of FE-BE coupling schemes for large-scale problems with fluid-structure interaction, Int. J. Numer. Meth. Engng.77 (2009) 664-688.

[18] S. Schneider. FE/FMBE coupling to model fluid-structure interaction, Int. J. Numer. 
Methods Engng. 76 (2008) 2137-56.

[19] M. Fischer, L. Gaul, Fast BEM-FEM mortar coupling for acoustic-structure interaction, Int. J. Numer. Methods Engng. 62(12) (2005) 1677-90.

[20] D. Brunner, M. Junge, L. Gaul, Strong coupling of the fast multilevel multipole boundary element method with the finite element method for vibro-acoustic problems, Proceedings of the 14th International Congress on Sound and Vibration (ICSV14), Cairns, 2007.

[21] J. Feng, X. Zheng, H. Wang, et al, Low-Frequency Acoustic-Structure Analysis Using Coupled FEM-BEM Method, Math. Probl. Eng. 2013(2) (2013) 5002-5058.

[22] H. Djojodihardjo, Vibro-acoustic analysis of the acoustic-structure interaction of flexible structure due to acoustic excitation, Acta Astronaut. 108 (2015)129-145.

[23] G. R. Liu, S. S. Quek, in: Finite Element Method: A Practical Course, Butter-worth-Heinemann, Burlington, MA, 2003.

[24] W. Li, Y.B. Chai, M. Lei, G.R. Liu. Analysis of coupled structural-acoustic problems based on the smoothed finite element method (S-FEM), Eng. Anal. Bound. Elem. 42 (2014) 84-91.

[25] E. Li, Z.C. He, X. Xu, G.R. Liu, Hybrid smoothed finite element method for acoustic problems, Comput. Methods Appl. Mech. Engrg. 283 (2015) 664-688.

[26] F. Ihlenburg, I. Babuska. Finite element solution of the Helmholtz equation with high wave-number, part I: the h-version of the FEM, Comput. Math. Appl. 30 (9) (1995) 9-37.

[27] J.S. Chen, C.T. Wu, S. Yoon, Y. You, A stabilized conforming nodal integration for Galerkin mesh-free methods, Int. J. Numer. Meth. Engng. 50 (2001) 435-466.

[28] J.S. Chen, S. Yoon, C.T. Wu, Non-linear version of stabilized conforming nodal integration for Galerkin mesh-free methods, Int. J. Numer. Meth. Engng. 53 (2002) 2587-2615.

[29] G.R. Liu, A generalized gradient smoothing technique and the smoothed bilinear form for Galerkin formulation of a wide class of computational methods, Int. J. Comput. Methods, 5 (2008) 199-236.

[30] G.R. Liu, A G space theory and a weakened weak $\left(\mathrm{W}^{2}\right)$ form for a unified formulation of compatible and incompatible methods: Part I Theory, Int. J. Numer. Meth. Engng. 81 (2010) 1093-1126.

[31] G.R. Liu, A G space theory and a weakened weak $\left(\mathrm{W}^{2}\right)$ form for a unified formulation of compatible and incompatible methods: Part II applications to solid mechanics problems, Int. J. Numer. Meth. Engng. 81 (2010) 1127-1156.

[32] L. Y. Yao, D. J. Yu, X. Y. Cui, et al, Numerical treatment of acoustic problems with the smoothed finite element method, Appl. Acoust. 71(8) (2010) 743-753.

[33] X. Y. Cui, H. Feng, G. Y. Li, et al, A cell-based smoothed radial point interpolation method (CS-RPIM) for three-dimensional solids, Eng. Anal. Bound. Elem. 50 (2015) 474-485.

[34] W. Zeng, G. R. Liu, C. Jiang, , et al, An effective fracture analysis method based on the virtual crack closure-integral technique implemented in CS-FEM, Appl. Math. Model. 40 (2016) 3783-3800

[35] G.R. Liu, L. Chen, T. Nguyen-Thoi, K.Y. Zeng, G.Y. Zhang, A novel singular node-based smoothed finite element method (NS-FEM) for upper bound solutions of fracture problems, Int. J. Numer. Meth. Engng. 83 (2010) 1466-1497.

[36] L. Chen, Y.W. Zhang, G.R. Liu, H. Nguyen-Xuan, Z.Q. Zhang, A stabilized finite element method for certified solution with bounds in static and frequency analyses of piezoelectric structures. Comput. Methods Appl. Mech. Engrg. 241-244(2012), 65-81. 
[37] G. Wang, X. Y. Cui, G. Y. Li, Temporal stabilization nodal integration method for static and dynamic analyses of Reissner-Mindlin plates, Comput. Struct. 152 (2015) 125-41.

[38] G. Wang, X. Y. Cui, H. Feng, et al, A stable node-based smoothed finite element method for acoustic problems. Comput. Methods Appl. Mech. Engrg. 297 (2015) 348-370.

[39] G.R. Liu, T. T. Nguyen, K. Y. Lam, An edge-based smoothed finite element method (ES-FEM) for static and dynamic problems of solid mechanics, J. Sound Vib. 320 (2009) $1100-30$.

[40] X. Y. Cui, G.R. Liu, G. Y. Li, G. Y. Zhang, G. Zheng, Analysis of plates and shells using an edge-based smoothed finite element method, Comput. Mech. 45 (2010) 141-56.

[41] Z. C. He, G.R. Liu, Z. H. Zhong, S. C. Wu, G. Y. Zhang, A. G. Cheng, An edge-based smoothed finite element method (ES-FEM) for analyzing three-dimensional acoustic problems. Comput. Methods Appl. Mech. Engrg. 199 (2009) 20-33.

[42] W. Zeng, G. R.Liu GR, Y. Kitamura, H. Nguyen-Xuan, A three-dimensional ES-FEM for fracture mechanics problems in elastic solids, Eng. Fract. Mech. 114 (2013) 127-150.

[43] T. T. Nguyen, G.R. Liu R, K. Y. Lam, G. Y. Zhang, A face-based smoothed finite element method (FS-FEM) for 3D linear and geometrically non-linear solid mechanics problems using 4-nodete trihedral elements. Int. J. Numer. Meth. Engng. 78(3) (2009) 324-53.

[44] G. Wang, X. Y. Cui, Z. M. Liang, et al, A coupled smoothed finite element method (S-FEM) for structural-acoustic analysis of shells. Eng. Anal. Bound. Elem. 61 (2015) 207-217.

[45] X. Xu, Y.T. Gu, G.R. Liu, A hybrid smoothed finite element method (H-SFEM) to solid mechanics problems, Int. J. Comput. Methods, 10 (2013) 1-17.

[46] W. Zeng, G.R. Liu, C. Jiang, et al, A generalized beta finite element method with coupled smoothing techniques for solid mechanics. Eng. Anal. Bound. Elem. 73 (2016) 103-119

[47] W. Zeng, G. R. Liu. Smoothed Finite Element Methods (S-FEM): An Overview and Recent Developments, Arch. Computat. Methods. Eng. (2016). doi:10.1007/s11831-016-9202-3

[48] G. Wang, X. Y. Cui, G. Y. Li, Acoustic simulation using a novel approach for reducing dispersion error, Int. J. Numer. Meth. Fl. (2016). doi:10.1007/s11831-016-9202-3

[49] F. Fahy, P. Gardonio, Sound and structural vibration: radiation, transmission and response, 2nd ed, New York: Academic Press, 2007.

[50] M. Junge, M. Fischer, M. Maess, L. Gaul, Acoustic simulation of an idealized exhaust system by coupled FEM and fast multipole BEM, Proceedings of the Twelfth International Congress on Sound and Vibration (ICSV12), Lisboa, 2005.

[51] K. U. Bletzinger, M. Bischoff, E. Ramm, A unified approach for shear-locking-free triangular and rectangular shell finite elements, Comput. Struct. 75 (2000) 321-334.

[52] J. B. Keller, D. Givoli, Exact non-reflecting boundary conditions. J. Comput. Phys. 82 (1989) 172-92.

[53] I. Harari, K. Grosh, T. J. R. Hughes, et al, Recent developments in finite element methods for structural acoustics, Arch. Computat. Methods. Eng. 3(2) (1996) 131-309.

[54] D. Giljohann, M. Bittner, The three-dimensional DtN finite element method for radiation problems of the Helmholtz equation, J. Sound Vib. 212 (1998) 383-94.

[55] Y. B. Chai, W. Li, Z. X. Gong, T. Y. LI, Hybrid smoothed finite element method for two dimensional acoustic radiation problems, Appl. Acoust. 103 (2016) 90-12.

[56] G. Ben-Porat, D. Givoli, Solution of unbounded domain problems using elliptic artificial boundaries, Int. J. Numer. Meth. Bio. 11 (1995) 735-741. 
